Assessing the exercise response to a bronchodilator in COPD

\section{Assessing the exercise response to a bronchodilator in COPD: time to get off your bike?}

\section{D L Morgan, S J Singh}

\section{A comparison of exercise tests}

$\mathrm{P}$ rogressive irreversible airway obstruction is the defining characteristic of chronic obstructive pulmonary disease (COPD). However, people with COPD do not complain of airway obstruction but, more usually, about the associated restriction of physical activity. The disability resulting from COPD may also be compounded by peripheral muscle dysfunction that further limits exercise capacity. Traditionally, we have used tests of whole body exercise performance to reflect this process. However, as we learn more about COPD, it is revealed as a complex condition where apparently straightforward truths are neither pure nor simple. The relationship between airway function and exercise capacity is a good example of this complexity, since it may naively be expected that the decline in physical activity may mirror the progression of airway obstruction. Furthermore, it might also be reasonable to expect that an improvement in airway function may lead to a similar gain in physical activity. In fact, neither of these phenomena is necessarily observed. The relationship between static lung function and exercise capacity is not predictive in COPD and, in addition, the forced expiratory volume in $1 \mathrm{~s}\left(\mathrm{FEV}_{1}\right)$ alone cannot be used to judge disability. Furthermore, the effects of short-term improvements in airway function by bronchodilation do not reliably lead to improved exercise capacity. This is clearly a source of frustration-both for patients whose performance may not improve and for those who seek to demonstrate the benefits of a therapeutic intervention. There are several possible explanations for the apparent disconnection between changes in lung function and exercise performance. One explanation lies in understanding the nature of exercise capacity limitation in COPD, which may vary with the stage of the disease. Another factor may be the method chosen to assess activity limitation. We usually use formal, standardised whole body exercise tests as a proxy for domestic physical activity. However, not all exercise tests assess the same physiological activity and may not always be the most appropriate or sensitive investigation to demonstrate therapeutic change. Some of these factors are explored by Pepin and co-workers in this issue of Thorax (see page 291) where they begin to address the subject with the degree of sophistication that it deserves.'

Advancing airway obstruction in COPD is associated with exercise limitation, but the factors that limit maximal incremental (peak) performance may be different from those limiting endurance (constant load) exercise. In health, maximal capacity is usually defined by the oxygen uptake of the respiring muscle mass. This is generally the product of oxygen delivery and muscle mass. Neither ventilation nor gas exchange are normally factors in maximal limitation, although peripheral muscle fatigue may be a complication. In contrast, submaximal constant workload exercise is limited by factors that affect endurance capacity. In health these include substrate usage, the state of peripheral muscle conditioning, motivation and thermoregulation. As COPD develops, two further factors become evident. First, ventilatory limitation begins to impede the exercise response and maximal exercise may eventually be terminated prematurely through the phenomenon of dynamic hyperinflation, although this is not universal. ${ }^{2}{ }^{3}$ Second, deconditioned peripheral muscles may result in subjective or physiologically limiting fatigue. ${ }^{4}$ Both of these factors will clearly play a major role in the termination of maximal exercise and may also curtail endurance exercise if the relative workload is sufficient for them to develop. An endurance or constant workload exercise test is usually set at a proportion of the maximal performance. In health, the sustainable fraction of maximal capacity that can be maintained as an endurance task is of the order of $75 \%$ and can be improved by training or diet. Ironically, people with COPD can sustain a slightly higher fraction of their peak capacity because the latter may be artificially reduced by the ventilatory limitation. ${ }^{5}$

The exploration of exercise pathophysiology in COPD has naturally taken place in the laboratory where motorised treadmills or cycle ergometers have been used as the exercise testing platform. Such laboratory-based studies have provided invaluable insight into the exercise responses in COPD. However, there are details in the methodology of these explorations that have created some misconceptions now that exercise testing is routinely used as an outcome for therapeutic intervention. Most studies have used the cycle ergometer as the exercise platform for two reasons. First, the cycle ergometer is a stable and safe platform from which it is easier to take samples or perform measurements while exercising. Second, the ergometer provides a direct measurement of workload. However, cycling is not the same as walking, and the two platforms are not interchangeable. Cycling uses less muscle mass but may produce a greater lactate response because of the unfamiliar use of the leg muscles. ${ }^{6}$ For the last couple of decades it has been perceived wisdom that maximal exercise in COPD is limited as much by the legs as by dyspnoea. ${ }^{7}$ However, this does not fit with patients' descriptions of their symptoms where breathlessness predominates. More recently a repetition of the comparison of limiting factors in COPD suggests that, when a walking test is used, dyspnoea is indeed the limiting symptom. ${ }^{8}$

When exercise tests are used as an outcome measure for therapeutic interventions in COPD, it is important-when interpreting the response-to understand the nature of the test that is being used. Tests of maximal capacity may provide insights into pathphysiology but, in COPD, they are unlikely to change much with a therapeutic intervention. Constant workload or endurance tests, by contrast, are more likely to be responsive to treatment for two reasons: (1) the factors that limit endurance exercise are more amenable to intervention and (2) because even a minor change in maximal capacity will reduce the relative workload of the constant workload test and amplify the response. We have become used to seeing reliable improvements in exercise capacity following exercise training in COPD in the context of pulmonary rehabilitation. ${ }^{9}$ In this situation, small improvements occur in maximal capacity but much greater benefits are evident on tests of exercise endurance. ${ }^{10}$ However, demonstration of a similar improvement in exercise capacity following administration of a bronchodilator has been inconsistent. 
The lack of predictability of the effects of bronchodilators on exercise capacity was described a few years ago in a systematic review by Liesker et al. ${ }^{11}$ At that time, 33 suitable randomised, double-blind studies were available for inclusion, but only about half of the trials were able to show an improvement in physical performance following a bronchodilator. In the review the authors discuss the possible reasons for this inconsistency, which appeared to relate more to the assumptions about testing than the qualities of the specific bronchodilator. For an improvement to occur, there should first have been demonstration of a ventilatory limit that could be relieved by bronchodilator in sufficient dose. In many studies the limits were not identified and bronchodilator responses in airway function were small. The types of exercise tests were varied and, in some cases, the exclusion of learning effects by practice testing was not performed. The mixture of tests included maximal testing, steady state exercise and field walking tests. For the reasons explained earlier, some of these tests will lack sensitivity. However, there were suggestions that steady state exercise-particularly constant load cycle exercise-was the most sensitive approach. Since the publication of the systematic review, the evidence for the usefulness and sensitivity of constant load cycle testing at $50-80 \%$ of peak workload has been growing. At the same time, we have learnt more about the nature of dynamic hyperinflation as a limiting factor and its relationship to endurance time. The reliability of cycle endurance testing has been demonstrated, and it has now been used in a number of trials-mainly by O'Donnell's group in Canada-that have clearly shown benefit for all classes of bronchodilator. ${ }^{12-16}$

Although cycle endurance testing appears to be a sensitive investigation for change following a bronchodilator, it does not appear to be the whole story. There are good reasons to believe that walking is a more appropriate exercise task to demonstrate benefit than cycling. This is because improved walking ability is a task and ambition that is more relevant to the lives and aspirations of people with COPD ${ }^{17}$ Also, we know that cycling places less metabolic demand on subjects than walking but results in more chance of leg fatigue. ${ }^{18}$ Consequently, the demonstration of a bronchodilator effect that may release some of the ventilatory constraint may be overshadowed by the impact of cycling on the legs. This hypothesis was tested recently in a study which compared the effects of bronchodilation on cycle endurance time and the 6 min walking test $(6 \mathrm{MWT}){ }^{19}$ The Japanese authors found that the cycle testing was more sensitive, seemingly vindicating the prominent position of cycle endurance testing. However, this examination has been extended by another Canadian group who sought to explain the apparent contradictions. In two papers, Pepin et al have argued that the type of walking test that is used may influence the result. ${ }^{18}$ At present, there are three main field walking tests in common use-the 6MWT and the incremental and endurance shuttle walks. ${ }^{10} 2021$ The 6MWT is probably the most popular investigation because of its simplicity, relevance to daily life, widespread use and availability of reference values. However, it is not physiologically pure because it is unpaced and, unless appropriately conducted, may be subject to learning and motivation effects. Furthermore, it is a physiological hybrid test that may produce erratic metabolic demand that may include peak or endurance performance. ${ }^{22}$ The shuttle walk tests are strictly paced and were developed to provide field equivalents of laboratory incremental and constant workload tests. In the original incremental shuttle walk test the pace across the $10 \mathrm{~m}$ course is increased every minute to a symptom limit. In the endurance shuttle walk the pace is constant at a proportion (80-85\%) of a prior incremental test and the patient continues until exhaustion. The endurance shuttle walk test is the most likely candidate to demonstrate sensitivity, and this has been the subject of the recent explorations by Pepin et al. In the first paper the authors explored the sensitivity of the endurance shuttle walk test to acute bronchodilation with ipratropium bromide compared with endurance cycle testing. ${ }^{8}$ The patients had a significant bronchodilator response to $\mathrm{FEV}_{1}$ and, in this double-blind crossover design, showed that cycle testing was not as sensitive as endurance shuttle walking. This was in part explained by the negative effect of the quadriceps fatigue that developed after cycling. The paper published in this issue of Thorax naturally completes the circle of investigation by comparing the sensitivity of carefully conducted 6MWT and endurance shuttle walk tests. ${ }^{1}$ In this case the study is accompanied by ambulatory metabolic measurements and post-exercise quadriceps twitch measurements. Despite demonstrating an acute improvement in $\mathrm{FEV}_{1}$, the 6MWT did not record an improvement in exercise capacity that was evident on the endurance shuttle test. Quadriceps fatigue was not present on either test, and the authors conclude that the endurance shuttle walk is the most responsive investigation available to record the exercise benefits of bronchodilation.
So where does all this leave us? The simple concept is that small improvements in airway function may, in most cases, translate into meaningful benefits in exercise capacity by reduction of dynamic hyperinflation. This benefit is best described by a test of whole body endurance capacity that uses a task that does not result in quadriceps fatigue through unfamiliar activity or require an equipped exercise laboratory. The endurance shuttle walk test seems to fit the bill in this respect, at least in countries where cycling is a minority activity. However, a note of caution is required and the field still requires clarification. We may now understand what is required in an assessment tool, but still need to acknowledge the context of the testing. Most bronchodilator studies have been performed in response to the acute administration of a drug. It is possible, though, that even removal of a ventilatory limit may not result in an immediate improvement in exercise performance because this simply uncovers peripheral muscle dysfunction. Patients may then require time or training to make use of the greater ventilatory capacity. The additional legitimising benefits of bronchodilation on physical training in this respect have already been demonstrated. ${ }^{23}$ We have learnt a lot about COPD in recent years, and each discovery adds a layer of complexity to a condition that has previously been dismissed with oversimplification. It is unlikely that the progress of investigation in the area of the COPD exercise response will end at this point. For the time being, however, we should probably get off our bikes and start walking.

Thorax 2007:62:281-283.

doi: 10.1136/thx.2006.069195

\section{Authors' affiliations}

M D L Morgan, S J Singh, Institute for Lung Health, Department of Respiratory Medicine, University Hospitals of Leicester, Glenfield Hospital, Leicester LE3 9QP, UK

Correspondence to: $\operatorname{Dr} M$ D L Morgan, Institute for Lung Health, Department of Respiratory Medicine, University Hospitals of Leicester, Glenfield Hospital, Leicester LE3 9QP, UK; mike.morgan@uhl-tr.nhs.uk

Conflict of interest: None declared

\section{REFERENCES}

1 Pepin V, Brodeur J, Lacasse Y, et al. Six-minute walking versus shuttle walking: responsiveness to bronchodilatation in chronic obstructive pulmonary disease. Thorax 2007:62:291-8.

2 Aliverti A, Stevenson N, Dellaca RL, et al. Regional chest wall volumes during exercise in chronic obstructive pulmonary disease. Thorax 2004;59:210-6.

3 Aliverti A, Rodger K, Dellaca RL, et al. Effect of salbutamol on lung function and chest wall volumes 
at rest and during exercise in COPD. Thorax 2005;60:916-24.

4 Man WD, Soliman MG, Gearing J, et al. Symptoms and quadriceps fatigability after walking and cycling in chronic obstructive pulmonary disease. Am J Respir Crit Care Med 2003; 168:562-7

5 Punzal PA, Ries AL, Kaplan RM, et al. Maximum intensity exercise training in patients with chronic obstructive pulmonary disease. Chest 1991; 100:618-23.

6 Mathur RS, Revill SM, Vara DD, et al. Comparison of peak oxygen consumption during cycle and treadmill exercise in severe chronic obstructive pulmonary disease. Thorax 1995;50:829-33.

7 Killian KJ, Leblanc P, Martin DH, et al. Exercise capacity and ventilatory, circulatory, and symptom limitation in patients with chronic airflow limitation. Am Rev Respir Dis 1992; 146:935-40.

8 Pepin V, Saey D, Whittom F, et al. Walking versus cycling: sensitivity to bronchodilation in chronic obstructive pulmonary disease. Am J Respir Crit Care Med 2005;172:1517-22.

9 Lacasse Y, Goldstein R, Lasserson TJ, et al. Pulmonary rehabilitation for chronic obstructive pulmonary disease. Cochrane Database Syst Rev 2006:(4):CD003793.

10 Revill SM, Morgan MD, Singh SJ, et al. The endurance shuttle walk: a new field test for the assessment of endurance capacity in chronic obstructive pulmonary disease. Thorax 1999:54:213-22.

11 Liesker JJ, Wiikstra PJ, ten Hacken $\mathrm{NH}$, et al. A systematic review of the effects of bronchodilators on exercise capacity in patients with COPD. Chest 2002;121:597-608

12 O'Donnell DE, Sciurba F, Celli B, et al. Effect of fluticasone propionate/salmeterol on lung hyperinflation and exercise endurance in COPD. hyperinflation and exercise
Chest 2006; 130:647-56

13 O'Donnell DE, Voduc N, Fitzpatrick M, et al. Effect of salmeterol on the ventilatory response to exercise in chronic obstructive pulmonary disease. Eur Respir J 2004;24:86-94.

14 O'Donnell DE, Fluge T, Gerken F, et al. Effects of tiotropium on lung hyperinflation, dyspnoea and exercise tolerance in COPD. Eur Respir J 2004; 23:832-40.

15 Oga T, Nishimura K, Tsukino M, et al. A comparison of the effects of salbutamol and ipratropium bromide on exercise endurance in patients with COPD. Chest 2003;123:1810-6.

16 van't HA, Gosselink R, Kwakkel G. Constant-load cycle endurance performance: test-retest reliability and validity in patients with COPD. J Cardiopulm Rehabil 2003;23:143-50.

17 Sewell L, Singh SJ, Williams JE, et al. Can individualized rehabilitation improve functional independence in elderly patients with COPD? Chest 2005; 128: 1194-200.

18 Palange $P$, Forte $S$, Onorati $P$, et al. Ventilatory and metabolic adaptations to walking and cycling in patients with COPD. J Appl Physiol 2000;88:1715-20.

19 Oga T, Nishimura K, Tsukino M, et al. The effects of oxitropium bromide on exercise performance in patients with stable chronic obstructive pulmonary disease. A comparison of three different exercise tests. Am J Respir Crit Care Med 2000;161:1897-901

20 Butland RJ, Pang J, Gross ER, et al. Two-, six-, and 12-minute walking tests in respiratory disease. BM (Clin Res Ed) 1982;284:1607-8.

21 Singh SJ, Morgan MD, Scott S, et al. Development of a shuttle walking test of disability in patients with chronic airways obstruction. Thorax 1992:47:1019-24.

22 Onorati P, Antonucci R, Valli G, et al. Non-invasive evaluation of gas exchange during a shuttle walking test vs. a 6-min walking test to assess exercise tolerance in COPD patients. Eur J Appl Physio 2003:89:331-6.

23 Casaburi R, Kukafka D, Cooper CB, et al. Improvement in exercise tolerance with the combination of tiotropium and pulmonary rehabilitation in patients with COPD. Chest 2005;127:809-17.

\section{Blanching the airways: steroid effects in asthma}

\section{Alan J Knox, Karl Deacon, Rachel Clifford}

\section{An important effect of steroids on angiogenesis in asthma}

$\mathrm{T}$ he vascular changes which occur in airways diseases such as asthma are starting to attract considerable attention from the respiratory research community. In addition to the vascular engorgement which occurs as part of the acute inflammatory process, several groups have demonstrated increased new vessel formation (angiogenesis) in chronic asthma. ${ }^{1-3}$ Not only does this occur in adult asthma, but recent studies suggest it is a prominent feature of childhood asthma, suggesting that vascular remodelling may occur relatively early in the asthmatic process. ${ }^{4}$ The increased airway wall thickening produced by the expanded vasculature causes enhanced airway narrowing on stimulation with constrictor agents, thereby contributing to bronchial hyperresponsiveness. Furthermore, the increased blood flow may increase inflammatory cell trafficking and exudation and transudation of cytokines and mediators and contribute to airway hyper-responsiveness by supporting the increased airway smooth muscle mass which is a key feature of asthma histopathology. ${ }^{5}$

There are a number of candidate angiogenic factors for these changes, perhaps the most important of which are vascular endothelial growth factor (VEGF) and angiopoietin-1, distinct molecules which act together at different stages of angiogenic processes in several biological systems. ${ }^{6-15}$ Other molecules with angiogenic potential found in the airways include fibroblast growth factor, ${ }^{10}$ angiogenin $^{10}$ and chemokines such as interleukin (IL)- $8^{16}$ and eotaxin. ${ }^{17}$ VEGF is subject to dynamic regulation while angiopoietin- 1 is less so, and the latter may contribute in a more permissive way to the remodelling process. A number of stimuli can increase VEGF release from lung cells including cigarette smoke, hypoxia and Thl and Th2 cytokines such as IL1 $\beta$, IL4 and IL13, remodelling cytokines such as TGF $\beta$ and IL6, and vasoactive mediators such as bradykinin and $\mathrm{PGE}_{2} \cdot{ }^{18-27}$ Autocrine production of $\mathrm{PGE}_{2}$ may mediate the effect of some of these agents, ${ }^{1827}$ and there is evidence from studies in mouse models to suggest that autocrine nitric oxide production may mediate some (but not all) of the effects of released VEGF in mouse asthma models. ${ }^{28}$ Endogenous angiostatic molecules such as endostatin and angiopoietin-2 exert a brake on this process, and the dynamic interplay between these and pro-angiogenic molecules helps shape repair and remodelling. ${ }^{29}$

Interestingly, recent studies in vitro with rhinovirus have shown that infection increases VEGF $^{30}{ }^{31}$-but not angiopoietin ${ }^{30}$-release, suggesting a mechanism whereby recurrent viral airway infections might contribute to airway remodelling in a cyclical manner. In mouse asthma models, airway VEGF is increased and VEGF receptor inhibitors inhibit cellular influx as well as inhibiting airway hyper-responsiveness and reducing microvascular leakage, ${ }^{32}$ consistent with VEGF having an important deleterious effect in asthma. In these and other studies, ${ }^{15}$ VEGF appears to regulate inflammatory processes as well as remodelling, which suggests that it is a complex multifunctional molecule with a wide repertoire of effects. There also appears to be a close relation between VEGF and matrix degradation which probably reflects the fact that establishment of 